\title{
Comparison of the McGrath MAC video laryngoscope and the Pentax Airwayscope during chest compression: a manikin study
}

Atsushi Kotera ${ }^{1 *}$, Hiroki Irie ${ }^{1}$, Shinsuke Iwashita ${ }^{1}$, Junichi Taniguchi ${ }^{1}$, Shunji Kasaoka ${ }^{1}$ and Yoshihiro Kinoshita ${ }^{2}$

\begin{abstract}
We tested the utility of the McGrath MAC ${ }^{\circledast}$ (McG) video laryngoscope during chest compression compared with the Pentax Airwayscope ${ }^{\circledast}$ (AWS). We recruited 59 participants into the simulation study. The difference in the time to intubation (TI [sec]) between without and with chest compression was significant for the AWS attempts (median 13 , range $6-28$ vs. median 15 , range $6-72, p=0.0247$ ) but not significant for the McG attempts (median 16, range 6-75 vs. median 16, range 6-71); however, the difference of the TTls is not serious clinically. The utility of the two devices during chest compressions is almost similar although their characteristics are different.
\end{abstract}

Keywords: McGrath MAC, Pentax AirwayScope, Chest compression

\section{Introduction}

The Pentax Airwayscope (AWS; Pentax, Tokyo, Japan) can improve the view of the glottis during chest compression $[1,2]$. However, the AWS occasionally contacts the arm of the chest compressor because of its large body [3]. We constructed a hypothesis that a compact device could facilitate the intubation attempt during chest compression. The McGrath $\mathrm{MAC}^{\ominus}$ video laryngoscope (McG; Covidien, Tokyo, Japan) is a new developed compact device. Here, we compared the utility of the two devices.

\section{Findings}

We recruited the 59 participants (51 medical university students and eight medical vocational college students) into the simulation study. We used the Airman (Laerdal, Sentrum and Stavanger, Norway) as the manikin model. The participants performed intubation attempts in the following order: AWS without chest compression, AWS with chest compression, McG without chest compression, and McG with chest compression. Chest compression was performed by an Advanced Cardiac Life Support provider.

\footnotetext{
* Correspondence: w03tfpd922@hi3.enjoy.ne.jp

'Department of Emergency and General Medicine, Kumamoto University Hospital, 1-1-1 Honjo, Chuo-ku, Kumamoto City, Kumamoto Prefecture 860-8556, Japan

Full list of author information is available at the end of the article
}

We used an endotracheal tube (ETT; Portex, St. Paul, MN, USA) with an internal diameter of $7.0 \mathrm{~mm}$.

We measured the time to tracheal intubation (TTI) of each intubation attempt. For the McG attempts, the TTI was defined as the duration from grasping the device to removing the metal stylet from the ETT. For the AWS attempts, the TTI was defined as the duration from grasping the device to removing the blade from the manikin's mouth. We defined 'failed tracheal intubation' as either esophageal intubation or exceeding the time limit of $90 \mathrm{~s}$ for the attempt. We used the Mann-Whitney $U$ test to test for differences in the TTIs, and we considered $p$ values $<0.05$ significant.

The TTIs for each device are shown (Figure 1). The difference in the TTI (sec) between without and with chest compression was significant for the AWS attempts (median 13, range $6-28$ vs. median 15 , range $6-72$, $p=0.0247)$ but not significant for the McG attempts (median 16, range 6-75 vs. median 16, range 6-71). Two participants failed the intubation attempt in the McG attempts in each situation, and one participant in the AWS attempts in a situation with chest compression (Figure 1).

In general, in attempts with video laryngoscopes, the relative positions of the glottis and ETT in a monitor are stable during chest compression $[4,5]$. However, the 


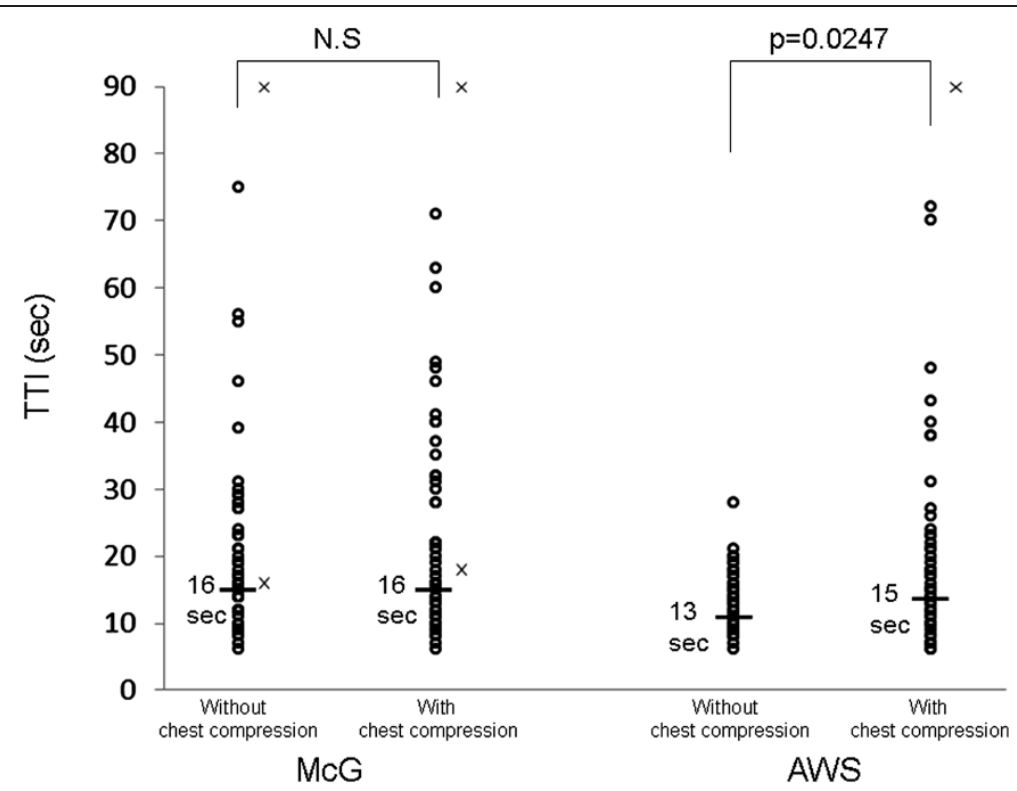

Figure 1 Scatter graph of TTI in all of participants using each device without and with chest compression. The thick bar indicates the median TTI. Open circles indicate successful tracheal intubations, and x indicates a failed tracheal intubation.

characteristics of each video laryngoscope are different and each device has its own advantages and disadvantages.

The distance from the device to the arm of the chest compressor differed between the two devices (Figure 2). In the present study, 42 of the 59 participants inserted

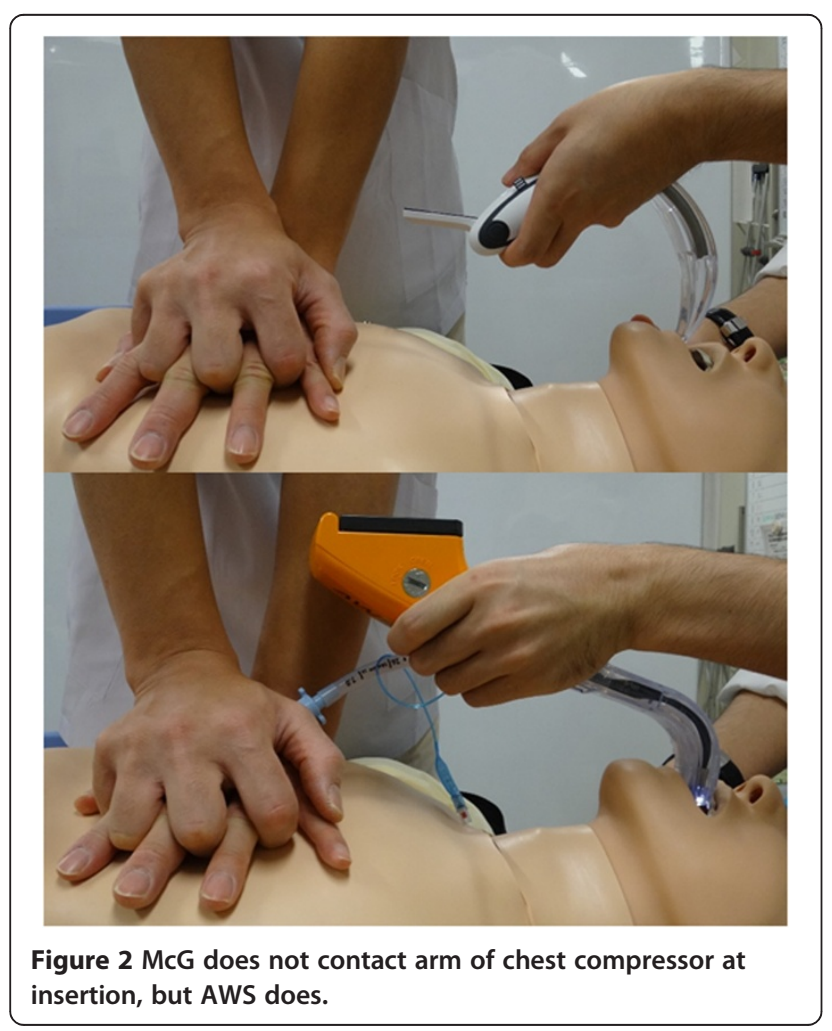

the AWS obliquely into the manikin's mouth in order to not contact the arm of the chest compressor. The oblique insertion of the AWS took more time. Conversely, the operator could insert the McG in a straight manner because of its compact body. The AWS's large body may contribute to the prolonged TTIs during chest compression. However, the difference of the TTIs is $2 \mathrm{~s}$, and that is not serious in a clinical situation. Additionally, there was no significant difference in the TTIs with chest compression in the attempts using the two devices. Considering our data, a compact device may not always facilitate the intubation attempt during chest compression.

The present study has several limitations. First, the manikin model cannot reproduce the precise intubation conditions of real patients. Second, chest compressions on a manikin model cannot duplicate CPR on real patients. Third, the participants are students who do not have enough skills needed for tracheal intubation. Fourth, the present study is not a randomized crossover study, and improvement in the participants' skills as each attempt progressed may have occurred.

Competing interests

All authors declare no financial or non-financial competing interests.

\section{Authors' contributions}

AK conceived the study, analyzed the results, and drafted the manuscript. HI contributed to the study design. SI and JT participated in the data collection. SK critically reviewed the manuscript and offered guidance. YK contributed to the study design and helped draft the manuscript. All authors reviewed this manuscript and approved the final submitted version. 


\section{Acknowledgements}

We thank all of the staff of the Department of Emergency and General Medicine and the Department of Intensive Care Medicine, Kumamoto University Hospital. We also acknowledge the participants in the present study.

\section{Author details}

${ }^{1}$ Department of Emergency and General Medicine, Kumamoto University Hospital, 1-1-1 Honjo, Chuo-ku, Kumamoto City, Kumamoto Prefecture 860-8556, Japan. ${ }^{2}$ Department of Intensive Care Unit, Kumamoto University Hospital, 1-1-1 Honjo, Chuo-ku, Kumamoto City, Kumamoto Prefecture 860-8556, Japan.

Received: 11 December 2013 Accepted: 12 February 2014

Published: 6 March 2014

\section{References}

1. Su YC, Chen CC, Lee YK, Lin KJ: Comparison of video laryngoscopes with direct laryngoscopy for tracheal intubation: a meta-analysis of randomized trial. Eur J Anaesthesiol 2011, 28:788-795.

2. Burdett E, Anderson DJ, Makepeace J, Bassett PA, Clarke SG, Mitchell V: Randomized controlled trial of the A.P Advance, McGrath, and Macintosh laryngoscope in normal and difficult intubation scenarios: a manikin study. Br J Anaesth 2011, 107:983-988.

3. Kim YM, Kim JH, Kang HG, Chung HS, Yim HW, Jeong SH: Tracheal intubation using Macintosh and 2 video laryngoscopes with and without chest compressions. Am J Emerg Med 2011, 29:682-686.

4. Suzuki A, Toyama Y, Katsumi N, Kunisawa T, Sasaki R, Hirota K, Henderson JJ, Iwasaki H: The Pentax-AWS ${ }^{\circledR}$ rigid indirect video laryngoscope: clinical assessment of performance in 320 cases. Anaesthesia 2008, 63:641-647.

5. Shin DH, Choi PC, Han SK: Tracheal intubation during chest compression using Pentax-AWS ${ }^{\oplus}$, GlideScope ${ }^{\oplus}$, and Macintosh laryngoscope: a randomized crossover trial using a mannequin. Can J Anesth 2011, 58:733-739.

doi:10.1186/2052-0492-2-18

Cite this article as: Kotera et al: Comparison of the McGrath MAC video laryngoscope and the Pentax Airwayscope during chest compression: a manikin study. Journal of Intensive Care 2014 2:18.

\section{Submit your next manuscript to BioMed Central and take full advantage of:}

- Convenient online submission

- Thorough peer review

- No space constraints or color figure charges

- Immediate publication on acceptance

- Inclusion in PubMed, CAS, Scopus and Google Scholar

- Research which is freely available for redistribution 